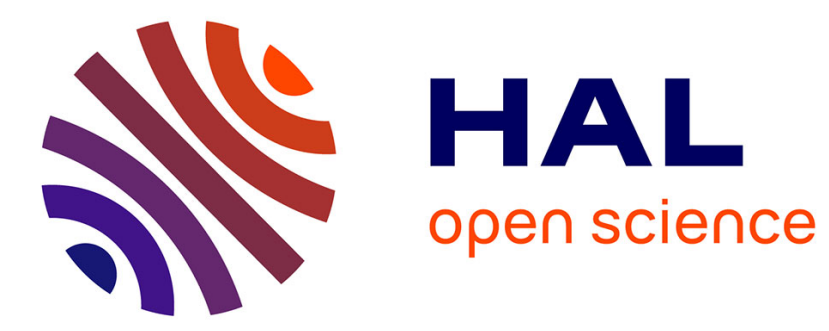

\title{
La dynamique de l'environnementalisme en France
}

François Facchini, Benjamin Michallet

\section{To cite this version:}

François Facchini, Benjamin Michallet. La dynamique de l'environnementalisme en France. Entreprendre \& Innover, 2017, 32 (1), pp.8-23. 10.3917/entin.032.0008 . hal-01678947

\section{HAL Id: hal-01678947 https://hal.science/hal-01678947}

Submitted on 11 Nov 2021

HAL is a multi-disciplinary open access archive for the deposit and dissemination of scientific research documents, whether they are published or not. The documents may come from teaching and research institutions in France or abroad, or from public or private research centers.
L'archive ouverte pluridisciplinaire HAL, est destinée au dépôt et à la diffusion de documents scientifiques de niveau recherche, publiés ou non, émanant des établissements d'enseignement et de recherche français ou étrangers, des laboratoires publics ou privés. 


\section{LA DYNAMIQUE DE L'ENVIRONNEMENTALISME EN FRANCE}

\section{François Facchini, Benjamin Michallet}

De Boeck Supérieur | «ntreprendre \& Innover »

$2017 / 1 n^{\circ} 32$ | pages 8 à 23

ISSN 2034-7634

ISBN 9782807391024

DOI 10.3917/entin.032.0008

Article disponible en ligne à l'adresse :

https://www.cairn.info/revue-entreprendre-et-innover-2017-1-page-8.htm

Distribution électronique Cairn.info pour De Boeck Supérieur.

(C) De Boeck Supérieur. Tous droits réservés pour tous pays.

La reproduction ou représentation de cet article, notamment par photocopie, n'est autorisée que dans les limites des conditions générales d'utilisation du site ou, le cas échéant, des conditions générales de la licence souscrite par votre établissement. Toute autre reproduction ou représentation, en tout ou partie, sous quelque forme et de quelque manière que ce soit, est interdite sauf accord préalable et écrit de l'éditeur, en dehors des cas prévus par la législation en vigueur en France. Il est précisé que son stockage dans une base de données est également interdit. 


\section{La dynamique de l'environnementalisme en France}

> François Facchini

> Benjamin Michallet

\section{Résumé}

Cet article mobilise la théorie de l'entrepreneur politique pour décrypter la diversité du recours aux problématiques environnementales dans les programmes des grandes familles politiques françaises. L'homme politique comme entrepreneur puise chez les intellectuels et les scientifiques un argumentaire rhétorique, qu'il diffuse aux électeurs à travers son programme politique. Les partis politiques jouent ainsi un rôle de caisse de résonance, en mobilisant les innovations idéelles à des fins politiques. En France, quatre grandes familles d’idéologie environnementale sont principalement mobilisées : l'environnementalisme anti-lumière, l'écologie politique, l’environnementalisme interventionniste et l’écologie de marché.

\section{Les points forts}

- Le poids de l'environnement dans le programme d'un parti est contingent à l'engagement d'un entrepreneur politique. La forme de l'environnementalisme choisi par le parti dépend d'un critère logique de cohérence idéologique.

- La part des mots consacrés à l'environnement dans le programme des partis verts atteint son maximum, soit 25 \% pour le parti Les Verts, en 1997, et ne cesse de baisser depuis. Cette part ne dépasse pas $6 \%$ pour les grands partis de gouvernement (PS et LR anciennement UMP).

- Ce n'est pas le parti vert qui a le premier popularisé l'écologie politique. C'est le parti communiste, à la fin des années 1950, et la droite dans une moindre mesure, à partir de 1968. 
$D_{s}^{a}$ ans un monde où le futur n'est pas la simple reproduction du passé, il y a une place pour l'innovation et l'entrepreneur. L'entrepreneur, en effet, est l'agent du changement. Il est celui qui transgresse et provoque ainsi la réorientation des ressources sur le marché. En politique, cette même définition de l'entrepreneur peut être retenue. Mais au lieu d'être l'agent du changement dans la hiérarchie des prix relatifs, l'entrepreneur politique ${ }^{1}$ est l'agent du changement institutionnel. Il est celui qui change le droit et le contenu des politiques publiques ${ }^{2}$. Il est celui qui crée des politiques innovantes et inattendues $^{3}$.

Une action politique est intentionnelle. L'intention est guidée par une fin que l'on peut qualifier d'idéale, qui elle-même est une réponse à un besoin de progrès, d'amélioration. Il cherche selon les cas à rendre un monde injuste juste et un monde inefficace efficace. L'action politique débute, pour cette raison, par la perception d'un problème: injustice ou situation défaillante. Elle se prolonge, ensuite, par la définition de solutions et d'une stratégie précise de mise en œuvre. Innover en politique signifie donc identifier un problème avant les autres, définir une solution et la mettre en œuvre. Cet article utilise l'histoire de l'environnementalisme en France pour illustrer ces trois étapes de l'innovation politique et montrer le rôle essentiel que joue l'entrepreneur politique dans

1 Facchini, F. (2006), "L'entrepreneur politique et son territoire ", Revue d'économie régionale et urbaine, 2, 263-280.

2 Weber, M. (2004), Euvres politiques (1895-1919), Paris, Albin Michel.

3 Riker, W. H. (1986), The art of political manipulation (Vol. 587), New Haven, Conn.: Yale University Press; Weissert, C.S. (1991), "Policy entrepreneurs, policy opportunists, and legislative effectiveness", American Politics Quarterly, 19(2), 262-274. la diffusion d'un idéal politique, le plus souvent identifié par un intellectuel ou un agent du monde académique. Il s'agit ainsi de comprendre comment l'invention du problème environnemental se transforme grâce au programme des partis politiques en innovation politique.

L'environnementalisme se prête bien à cette histoire parce qu'initialement la pollution n'est pas un problème. Jusqu'au début du XIXe siècle, en effet, "le sale, le malsain, l'infectieux, le corrompu ou simplement la puanteur sont les catégories qui permettent de penser ce que nous nommons aujourd'hui la pollution ${ }^{4}$. La foi dans le progrès amenait à penser ces pollutions seulement comme des effets regrettables, mais secondaires de l'industrialisation ${ }^{5}$. La pollution existait mais elle n'était pas perçue comme problématique. On en veut pour preuve le décret de 1810 sur les établissements insalubres, qui est généralement présenté comme la première décision censée lutter contre la pollution. Ce décret est, en fait, un moyen pour les grandes industries polluantes de se soustraire à la règle de responsabilité qui impose à chaque riverain de payer les dommages qu'il inflige à autrui ${ }^{6}$. La pollution ne devient un problème que parce que les sphères académiques et intellectuelles commencent à collecter un certain nombre d'informations qui montrent l'effet néfaste des pratiques industrielles sur la santé, la beauté des paysages, la moralité de nos rapports aux animaux, etc. ${ }^{7}$. Ces intellectuels transforment l'in-

4 Fureix, E. et larrige, F. (2015), La modernité désenchantée : relire l'histoire du XIXe siècle français, Paris, La Découverte, p. 91.
5 lbid., p. 91, note 5.
6 Ibid., p. 93, note 5 .
7 II existe certaines discussions à ce sujet, comme
le souligne Grégory Quenet. Les préoccupations 
salubre en pollution et font de l'industrialisation un problème. Ces problèmes ne deviennent politiques que lorsque les hommes politiques s'en saisissent et les mettent à l'agenda politique ${ }^{8}$. L'entrepreneur politique est ainsi l'agent qui fait d'une innovation intellectuelle une innovation politique. Il est l'agent qui identifie dans l'innovation intellectuelle un gain politique. Une fois ce gain identifié, son action change le monde des possibles politiques, car il fait de la pollution un problème et diffuse dans l'opinion avec les moyens qui sont les siens les arguments qui ont conduit les intellectuels à traiter de la pollution comme un réel problème.

Le monde des possibles politiques est défini comme l'ensemble des politiques publiques imaginées par les acteurs politiques pour répondre aux problèmes sociaux qu'ils ont identifiés comme prioritaires. Ce monde des possibles politiques dépend du monde des possibles idéologiques, autrement dit des dotations idéologiques de la société, qui sont elles-mêmes une fonction de l'activité des intellectuels et des agents de la sphère académique.

L'entrepreneur politique choisit donc parmi le monde des possibles environnementalistes une représentation de la relation homme-nature. Il lui donne ainsi une visibilité politique en l'inscrivant dans un programme politique qui sera la base sur laquelle il organisera son discours, sa campagne électorale et son action politique

environnementales seraient venues initialement des gens ordinaires. Cf. Quenet, G. (2014), Qu'est-ce que l'histoire environnementale? Seyssel, Champ Vallon, p. 256.

8 Stimson, I. A., Mackuen, M. B., \& Erikson, R. S. (1995), "Dynamic representation", American Political Science Review, 89(3), 543-565. une fois au gouvernement s'il est élu. La diffusion d'une idée, produite par un intellectuel ou un scientifique, est ainsi d'autant plus grande qu'elle est reprise par un entrepreneur politique qui est crédible et populaire. Un tel principe, s'il est connu des intellectuels et des scientifiques, peut les conduire à anticiper la demande des hommes politiques les plus influents et à créer une sorte de système de production d'idées qui produit de la notoriété et de la réputation pour les intellectuels et du discours et des politiques publiques pour les entrepreneurs politiques.

Le présent article expose de manière synthétique le déroulement du raisonnement sans développer chacune de ses étapes, avant de relater succinctement l'histoire des formes d'environnementalisme en France, autrement dit le monde des possibles idéologiques des rapports de l'homme à la nature. Il propose ensuite une histoire de la diffusion des idéologies dans le corps social via le verdissement des programmes politiques, tel qu'il peut être observé avec le Manifesto Project. II montre enfin comment le succès d'une forme d'environnementalisme dépend du poids électoral du parti qui la reprend. La contribution de cet article est donc double. Elle explique les programmes politiques par le monde des possibles idéologiques produits par les intellectuels et les scientifiques et la diffusion d'une innovation idéologique par l'écriture d'un programme qui joue le rôle d'une caisse de résonance.

9 Voir sur ce point: Kingdon, J. W. (1984), Agendas, alternatives, and public policies (Vol. 45), Boston, Mass.: Little, Brown, pp. 165-169 ; Klingemann, H. D., Hofferbert, R. I., \& Budge, I. (1994), Parties, policies, and democracy, Boulder-San Francisco-Oxford: Westview Press ; Laver, M. \& Garry, I. (2000), "Estimating policy positions from political texts", American Journal of Political Science, 44(3), 619-634. 


\section{L'histoire des écologies politiques en France}

L'innovation intellectuelle dans notre histoire correspond à l'invention dans la théorie schumpétérienne de l'innovation. L'intellectuel identifie dans la pollution un problème. Il est l'agent du changement de représentation parce qu'il propose une argumentation qui va augmenter les coûts de justification de l'affirmation selon laquelle la pollution n'est qu'un effet regrettable du progrès économique.

On peut dire que deux faits génèrent l'identification du problème environnemental: l'industrialisation et l'évolutionnisme darwinien. La nature n'est pas seulement un moyen ou une ressource. Elle est aussi le milieu où l'homme évolue et qui conditionne son existence et la qualité de cette dernière. Posé de cette manière, on comprend que l'écologie est une forme d'environnementalisme. L'homme prend, en effet, conscience que le productivisme, l'industrialisme, la mécanisation de toutes ses pratiques productives ainsi que sa consommation immodérée d'énergie fossile peuvent à terme menacer l'équilibre de la société. Cette prise de conscience caractérise toutes les formes d'environnementalisme. Ce qui les distingue, c'est la manière dont elles définissent l'équilibre.

De la critique de l'industrialisme à la critique du capitalisme, il n'y a qu'un pas. En fait, si l'industrialisation est la conséquence du capitalisme, on comprend que la pollution est la conséquence de l'industrialisation et donc du capitalisme. Il faudrait ainsi rompre avec le capitalisme actuel pour rétablir une relation équilibrée et de respect de l'homme à son environnement et à son milieu de vie. En réponse à ce diagnostic, l'émergence d'une écologie de marché (ou free ecology) propose des solutions de marché à l'ensemble des problèmes jugés importants par les environnementalistes ${ }^{10}$.

On comprend ainsi la pluralité des environnementalismes. Les intellectuels et les agents des sphères académiques ne vont pas tous avoir la même représentation du problème. Ils peuvent s'opposer sur son origine, sa cause, sur l'ampleur de ses conséquences, sur les solutions qu'il faut y apporter et sur la manière dont il faut agir pour mettre en œuvre ses solutions.

\section{Pour l'environnementalisme anti-} lumière, la cause profonde des relations de l'homme à la nature est la révolution libérale de 1789 et ses valeurs individualistes, matérialistes et marchandes. Car la société politique est organique, organisée autour de lois naturelles et d'une autorité forte et capable de représenter la nation. L'idéal, ici, est l'enracinement et l'ordre des communautés rurales. Son mot d'ordre pourrait être "la terre ne ment pas $»^{11}$. Ses principaux intellectuels sont Maurice Barrès et Charles Maurras à la fin du XIX siècle et au début du XX $X^{e}$ siècle ${ }^{12}$. Ces "lois naturelles" s'organiseraient autour d'un principe de sélection naturelle proche des théories de Spencer et du darwinisme social. L'autorité est appelée à être tenue par les forts qui

\footnotetext{
10 Une littérature importante étudie l'entrepreneur de marché comme solution aux problèmes environnementaux. On peut consulter à ce sujet le $n^{\circ} 22$ du Journal of Business Venturing paru en 2007.

11 Paxton, R. O. (1997), La France de Vichy 1940-1944, traduit de l'anglais par C. Bertrand, Paris: Seuil, coll. "Points Histoire ", pp. 253-262.

12 Nguyen, V. (1990), Aux origines de l'action française: intelligence et politique à l'aube du XXe siècle, Paris Fayard.
} 
nécessairement dominent les faibles. Le faible accepte d'autant mieux alors l'autorité des forts que ces derniers le protègent contre l'étranger et garantissent la survie du corps social dans son ensemble. Cet anti-modernisme trouve aujourd'hui un écho dans les travaux d'Alain de Benoist, de la Nouvelle Droite, du Groupement de recherche et d'études pour la civilisation européenne (GRECE), d’Éléments, ou encore du Recours aux forêts. Alain de Benoist publie en 2007 un livre intitulé Demain la décroissance ${ }^{13}$, qui crée ainsi un pont entre la critique de la Nouvelle Droite et la critique post-marxisme d'auteurs comme Alain Lipietz et Serge Latouche ou d'écologistes comme Michel Serres.

L'écologie politique est l'autre forme la plus connue d'environnementalisme. Le mot " écologie » est un néologisme forgé par le zoologiste allemand et militant de la cause eugéniste Ernst Haeckel. Ce zoologiste voulait en faire une branche des sciences de la nature. L'écologie se veut être une science de l'équilibre et de la préservation des milieux. Elle inspira, ensuite, les fondateurs de l'écologie politique et leur critique de la société moderne. Ces premiers penseurs de l'écologie en France sont des intellectuels comme l'essayiste Jacques Duboin, l'agronome René Dumont, les philosophes personnalistes Bernard Charbonneau et Jacques Ellul, le biologiste lean Dorst et/ ou l'économiste René Passet. Leurs discours reposent, comme l'environnementalisme anti-lumière, sur une critique du capitalisme et de ses conséquences:

13 de Benoist, A. (2007), Demain, la décroissance! Penser l'écologie jusqu'au bout, Paris: Édite. Dans ce livre, l'intellectuel de la Nouvelle Droite reprend le thème de l'impossibilité d'une croissance infinie dans un espace fini. Il traite du dérèglement climatique, de la dégradation de la nature, de la pollution, etc. l'épuisement des ressources naturelles, la mort des paysages, la destruction de la nature, la spécialisation des tâches provoquées par le progrès technique et le productivisme. L'écologie politique propose ainsi une critique du capitalisme dont les principales alternatives sont toutes les formes de socialisme: collectivisme, autogestion et social-démocratie. Le capitalisme est sans doute un bon système pour produire beaucoup, mais un tel objectif remet en cause la vie sur terre et défigure le visage de l'homme. L'homme est asservi à la machine et la machine est intimement liée à l'avènement du capitalisme qui ici est synonyme d'industrialisme. Les liens entre le socialisme associationniste et l'écologie reposent sur le goût des petites organisations contre les multinationales, en particulier celles qui exploitent le tiers-monde et détruisent le lien social.

L'écologie politique va donc naturellement, bien que plus tardivement, séduire les penseurs marxistes et trotskystes tels que le théoricien Ernst Mandel, le philosophe et théoricien Daniel Bensaïd, le philosophe André Gorz ou encore le psychologue social Serge Moscovici. En outre, ceux-ci théorisent et impulsent l'action écologiste révolutionnaire en opposition au mouvement réformiste, en dénonçant les positions défendues par Sicco Mansholt ${ }^{14}$. S'ils sont convaincus de l'urgence écologique, le combat qu'ils mènent représente un moyen supplémentaire de s'opposer au capitalisme $^{15}$ et permet à nouveau de pointer le caractère destructeur d'une société

14 Buton, P. (2012), "L'extrême gauche française et l'écologie ", Vingtième Siècle. Revue d'histoire, 113, 191-203 (ici p. 195).

15 Ibid., p. 197, note 1 . 
qui cherche la croissance de la production, le productivisme à tout prix. Il ne s'agit plus de dire que le capitalisme est un mauvais système de production, mais de remettre en cause le principe même d'une production de masse pour les masses $^{16}$.

\section{La posture technico-économique est une} réponse des courants interventionnistes traditionnels à l'écologie. Elle propose d'exploiter les bénéfices du productivisme sans ses coûts. Il ne s'agit pas de changer de modèle productif ou de revenir à des modèles plus proches des équilibres antérieurs à la Révolution française, mais de proposer des solutions techniques aux différents problèmes provoqués par le productivisme. Si le progrès technique asservit l'homme, il faut proposer des techniques qui laissent une plus grande place à la liberté de l'esprit. Si les techniques sont trop énergivores, il faut trouver des techniques moins consommatrices d'énergie. Si les hommes oublient de vivre pour travailler, il faut réguler le temps de travail. S'il y a de la pollution, c'est parce qu'il y a des externalités, qu'il faut internaliser par la mise en œuvre d'écotaxes. Historiquement l'économie de l'environnement ${ }^{17}$ naît dans cette forme d'environnementalisme. Le capitalisme est défaillant pour plusieurs raisons que sont le chômage, les inégalités, les externalités, l'existence de monopoles et plus généralement de situations de

16 La structuration des héritiers du marxisme autour de la question de l'écologie revêt en réalité une dimension beaucoup plus riche et complexe. Pour aller plus loin, on peut se référer à l'article de Philippe Buton (2016), "La gauche et la question écologique ", Revue française d'histoire des idées politiques, 44, 63-92.

17 Dorfman, R. et Dorfman N. F. (dir.) (1975), Économie de l'environnement, traduit de l'américain par D. Prompt et C. Gaston-Mathé, Paris: Calmann-Lévy (Economics of the Environment, New York: W.W. Norton, 1972). concurrence imparfaite, la présence de biens collectifs au sens de Samuelson ${ }^{18}$ et/ou d'asymétrie d'information ${ }^{19}$. Les dégradations de l'environnement relèvent des défaillances du marché et doivent être traitées comme telles. La théorie du développement durable est proche de cette vision technico-économique. Il s'agit de se développer sans compromettre la capacité des générations futures à répondre à leurs propres besoins (rapport Brundtland de 1987). Les aspects économiques, sociaux et environnementaux participent de cette conception politique du développement; les pouvoirs publics devant à tout moment agir pour maintenir l'équilibre de ces trois composantes du développement.

L'écologie de marché est la quatrième forme d'environnementalisme. Cette idéo-

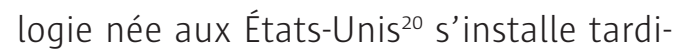
vement en France dans les années 1990. La publication en 1992 de l'ouvrage dirigé par Max Falque et Guy Millière intitulé Écologie et liberté: une autre approche de l'environnement ${ }^{21}$ marque les débuts de cette approche. L'argument principal est le suivant: l'épuisement des ressources naturelles et plus généralement la dégradation de l'environnement ne sont pas la conséquence du capitalisme, mais de son absence. De par sa prise de position pour la généralisation du régime

\footnotetext{
18 Biens caractérisés par le fait qu'on ne peut empêcher personne de les consommer et que la consommation d'une personne ne réduit pas la quantité disponible pour les autres. Ils sont dits non rivaux et non excluables.

19 Situation dans laquelle un coparticipant d'un échange bénéficie de plus d'information que les autres agents, lui conférant ainsi un avantage.

20 Batten, C. R. (1981), "Toward a Free Market in Forest Resources ", Cato Journal, 2(1), 501-517.

21 Falque, M. et Millière, G. (1992), Écologie et liberté une autre approche de l'environnement, Paris : Litec, coll. "Liberalia ".
} 
de propriété privée de toutes les ressources, régime permettant une gestion efficace des externalités, il apparaît également comme une solution à la dégradation de l'environnement. La surpêche, la surchasse, la dégradation des paysages, le déclin de la biodiversité, la pollution de l'eau et de l'air, autrement dit tous les problèmes soulevés par l'écologie politique ne trouvent pas leur origine dans le productivisme marchand, mais dans les défaillances des solutions publiques et/ou dans un régime de propriété qui n'incite pas à la protection et à un rythme d'exploitation soutenable des ressources rares $^{22}$.

Cette liste non exhaustive des mondes des possibles idéologiques environnementalistes est grossière, puisque chacun de ces courants recouvre une multitude de ramifications ${ }^{23}$. Malgré ses limites, elle permet de fixer les idées et de situer le discours politique dans chacun de ces courants sans placer mécaniquement le thème de l'environnement et de la nature à gauche de l'échiquier politique.

Face à la question environnementale, les intellectuels et les scientifiques de différentes sensibilités ont produit une argumentation, un discours qui sont plus ou moins compatibles avec l'idéologie politique des leaders de chaque grand parti. Il peut aussi inspirer l'engagement politique d'intellectuels ou de scientifiques qui peuvent juger qu'ils doivent eux-mêmes exposer leur représentation du monde afin de lui donner plus de visibilité et plus

22 On peut lire, pour une présentation accessible en français, Falque, M. (2015-2016), Propos écologiquement incorrects, tomes 1 et 2, Nice: Éditions Libre Échange, coll. "Intemporel".

23 On peut consulter à ce sujet le $n^{\circ} 44$ de la Revue française d'histoire des idées politiques, paru au second semestre 2016. de poids dans le corps social. En démocratie, en effet, c'est la loi du nombre du compte. Il faut donc que le maximum de citoyens partage votre vision du monde pour que cette dernière inspire les choix de politique publique et fonde l'évolution du droit.

\section{Le verdissement des programmes et l'entrepreneur politique}

Le principal instrument de diffusion des innovations politiques est le discours. Ce dernier informe le corps électoral de l'idéal du parti et de ses candidats. L'entrepreneur politique peut par calcul (intérêt) ou idéologie (sens de l'intérêt général) percevoir dans l'un de ces mondes des possibles idéologiques un gain politique. Il s'approprie alors une forme d'environnementalisme et en fait son idéal. II transforme une innovation intellectuelle en innovation politique. Il fait de l'environnement un enjeu politique. Il donne alors potentiellement à l'idéal environnementaliste la possibilité de changer les pratiques sociales par le haut (top-down) puisque la politique est par nature un mécanisme de transformation qui privilégie l'autorité sur les autres formes d'évolution.

La diversité des programmes politiques reflète alors en partie la pluralité des mondes des possibles idéologiques. Une idéologie, une innovation intellectuelle peuvent, en effet, ne jamais devenir une innovation politique. Une telle innovation ne se diffuse que par le bas (bottom-up). Dans ce cas, les acteurs changent leur pratique et le droit valide in fine cette évolution. L'entrepreneur politique n'est pas dans ce cas l'agent du changement. Il ne fait que l'accompagner. 


\section{Le Manifesto Project}

Le Manifesto Project produit une base de données sur le contenu des programmes politiques au moyen de méthodes d'analyses textuelles de "quasi-phrases". Les programmes politiques de plus de 1000 partis répartis dans cinquante pays depuis 1945 sont ainsi codés en 110 variables quantitatives. Ces variables correspondent à la part du programme politique (en \%) allouée par le parti observé à un thème particulier tel que la "référence positive à la protection de l'environnement". Ces données fournissent des informations uniques sur les préférences politiques des partis et sont de plus en plus utilisées en sciences politiques comme en sciences économiques. Au-delà de leur exhaustivité, leur principale qualité tient au fait qu'elles varient dans le temps et l'espace, offrant ainsi la possibilité de recourir à différentes modélisations selon les besoins des chercheurs. Ces données permettent notamment l'analyse de l'offre programmatique des partis, de la relation entre partis et votants, du rôle des partis au Parlement ou de la transformation des promesses politiques en politiques publiques. En 2003, le projet a reçu le prix de l'American Political Science Association (APSA) récompensant la meilleure base de données en politique comparative. Depuis 2009, le Manifesto Project bénéficie d'un financement de long terme de la part de la German Science Foundation dans le cadre du Manifesto Research on Political Representation (MARPOR) qui fait suite au Manifesto Research Group (MRG, 19791989) et au Comparative Manifesto Project (CMP, 1989-2009). https://manifestoproject.wzb.eu.

La base de données Manifesto-Project database (voir ci-dessus) permet de définir précisément le moment où l'environnementalisme entre dans les programmes politiques. Cette base code en effet les programmes de tous les grands partis français depuis 1945 et permet ainsi de dater à 1962 l'apparition de l'item
" environnement" dans le discours des partis. Ce seraient les partis d'extrême gauche (parti communiste) et dans une moindre mesure les partis de droite ${ }^{24}$ qui auraient été les premiers à percevoir dans l'environnementalisme un gain politique (figure 1). La figure 1 permet d'affirmer que l'environnementalisme trouve sa place initialement à l'extrême gauche, c'est-à-dire dans le programme du Parti communiste français (PCF) de la fin des années 1950 et à droite de manière significative après les événements de mai 1968. Ce résultat contredit l'idée souvent défendue par les politistes, selon laquelle les mouvements d'écologie politique auraient obligé les grands partis à introduire l'environnement dans leur programme politique ${ }^{25}$.

La figure 1 montre que les partis politiques ne donnent pas beaucoup de place à la thématique environnementale. La part des mots consacrés à ces thématiques est comprise en 0 et $8 \%$ des textes des programmes. C'est le deuxième enseignement plutôt surprenant de l'usage de cet indicateur de verdissement des programmes. Le troisième enseignement est qu'en dehors des partis verts, c'est l'extrême droite qui donne la plus grande importance à la thématique de l'environnement dans son programme aux législatives de 1997. Si l'on en croit les indicateurs quantitatifs du Manifesto Project, les extrêmes seraient donc les plus concernés par la question environnementale.

\footnotetext{
24 La réponse Gauche/Droite à la question environnementale est discutée par Neumayer (2004).

25 Voir sur ce point les travaux de Meguid, B. M. (2005). "Competition between unequals: The role of mainstream party strategy in niche party success ", American Political Science Review, 99(3), 347-359 ; Spoon, I. I., Hobolt, S. B., \& Vries, C. E. (2014), "Going green: Explaining issue competition on the environment ", European Journal of Political Research, 53(2), 363-380.
} 
Figure 1 : L'environnementalisme dans les programmes des partis politiques français de 1945 à 2012 (part de l’environnement en \% dans chaque programme)

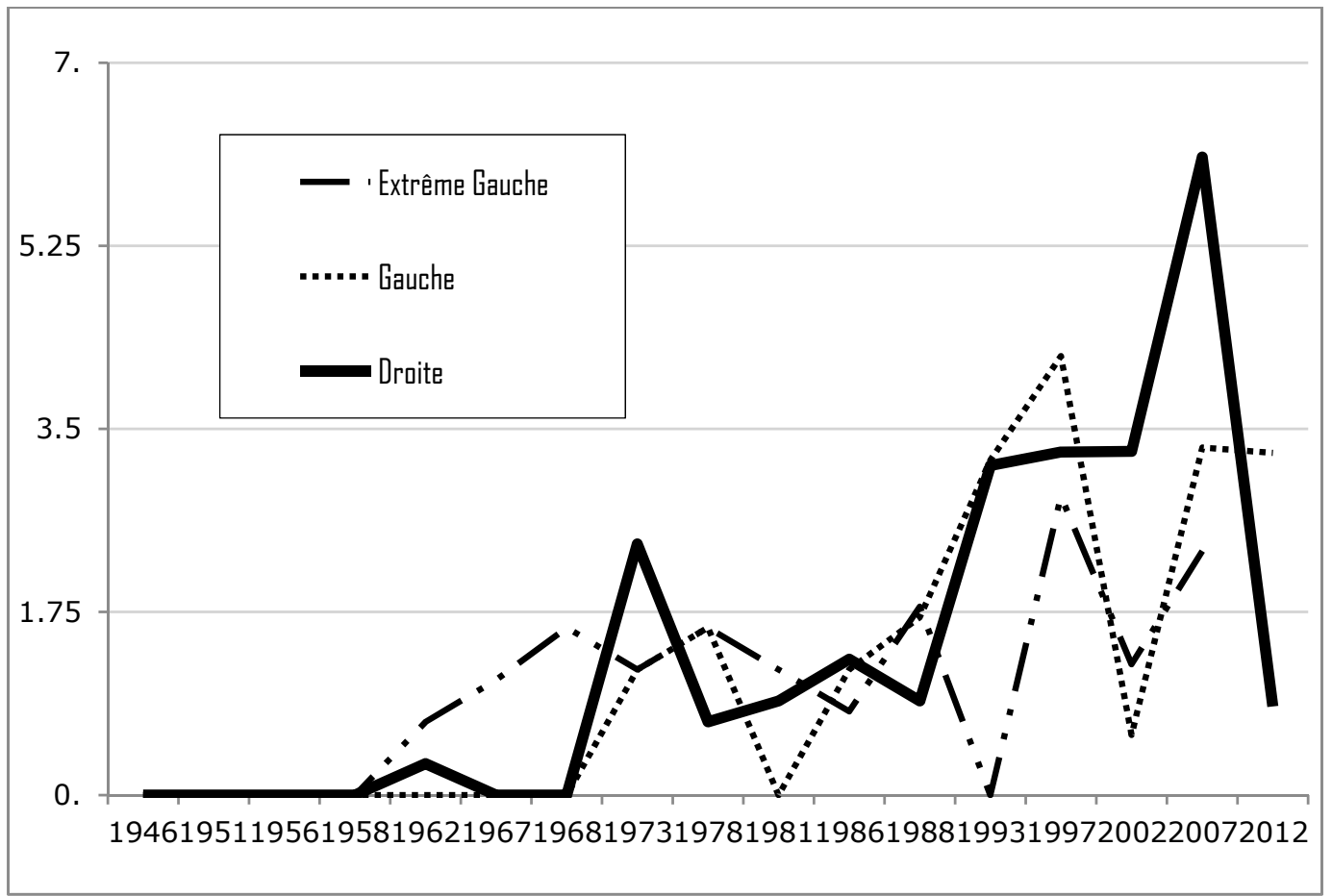

Source: Manifesto Project Data Set 2016b, disponible sur https://manifesto-project.wzb.eu.

En France, les partis verts restent néanmoins les principaux dépositaires de la question dans les programmes politiques. En effet, ils consacrent jusqu'à $25 \%$ (année 1997) de leur proposition programmatique à la question de l'environnement (figure 2). Cette proportion évolue néanmoins à la baisse, rappelant l'obligation pour un parti politique qui souhaite peser dans les débats nationaux et obtenir des élus, de proposer un programme plus généraliste. Même si initialement cela a été rappelé, l'écologie politique se veut être une alternative globale aux idéologies politiques qui partagent l'idéologie du progrès économique et technique.

Quelques articles encore rares cherchent à rendre compte de l'évolution de cette thématique environnementale dans les programmes des grands partis. Une première explication consiste à insister, comme mentionné précédemment, sur l'effet de la formation de partis verts et notamment en 1974 de la candidature de René Dumont à l'élection présidentielle. Ce serait la concurrence avec un parti vert que les partis de gouvernement redouteraient ${ }^{26}$. Les économistes, sans surprise, insistent plus, de leur côté, sur les grandeurs macroéconomiques, mais également sur l'adhésion à l'Union européenne, qui est négativement corrélée à l'offre environnementale des partis politiques des pays de l'Union. Cela signifie que le verdissement des programmes

26 Persico, S. (2015), " En Parler ou pas ? La place des enjeux environnementaux dans les programmes des grands partis de gouvernement ", Revue française de science politique, 65(3), 405-428. 
Figure 2 : Part du programme du Parti Vert consacré à l'environnement (1993-2012)

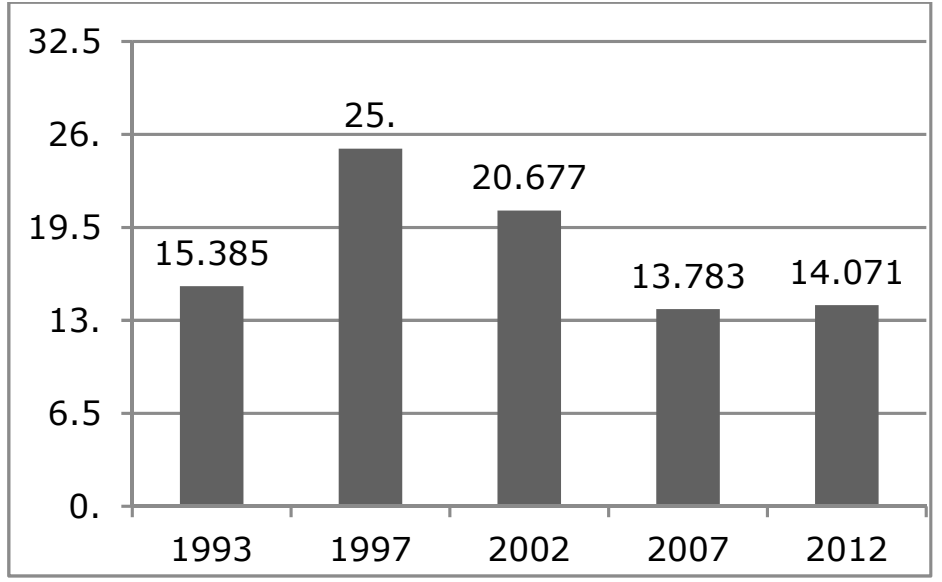

Source: Manifesto Project Data Set 2016b, disponible sur https://manifesto-project.wzb.eu.

n'est que la conséquence des injonctions des instances supranationales ${ }^{27}$.

\section{Les programmes}

\section{politiques comme véhicule de diffusion des possibles environnementalistes}

Les programmes des partis politiques ne se distinguent pas seulement par l'importance qu'ils donnent à la question environnementale. Ils se différencient aussi par le type d'écologie qu'ils choisissent. La lecture attentive de la dimension environnementale des partis politiques français permet d'associer un parti à une forme d'environnementalisme. Ils donnent ainsi à chaque innovation intellectuelle, à chaque type d'idéologie politique, son poids dans le corps électoral.

27 Michallet, B., Gaeta, G. L., \& Facchini, F. (2017), "Greening up or not? The determinants of political parties' environmental concern: an empirical analysis based on European data (1970-2008) ", forthcoming, in Land Use Policy.
L'entrepreneur politique identifie dans une innovation intellectuelle un gain. Le parti l'inscrit dans son programme et en fait une innovation politique. L'innovation intellectuelle se diffuse alors dans le corps électoral non plus par le bas (lecture, médias, discussion quotidienne, boucheà-oreille), mais par le haut (campagne électorale). L'efficacité de ce processus peut être mesurée par le pourcentage de voix aux élections reçues par le candidat. Ce poids électoral mesure l'impact de chaque forme d'environnementalisme dans le corps politique de la nation. Cela a deux conséquences. Une idée est d'autant plus populaire qu'elle est prise en charge par un parti politique puissant et capable de la populariser grâce à ses réseaux au Parlement, dans le corps électoral et dans les médias. La sélection des idéologies dans le corps politique, autrement dit les théories les plus partagées, ne sont pas nécessairement les plus scientifiques ou les plus cohérentes. Ce sont les idéologies sélectionnées par les hommes politiques à des fins électoralistes ou idéalistes. Il faut 
alors qu'elles répondent à leur conviction (rationalité axiologique) et à leur intérêt (rationalité instrumentale) et qu'elles correspondent à la connaissance qu'ils ont accumulée sur la question environnementale.

Le Front National reprend une forme plus ou moins fidèle d'environnementalisme anti-lumière ou conservateur. L'environnementalisme entre dans le programme du parti via Bruno Mégret qui y diffuse les idées de la Nouvelle Droite (François, 2016, p. 18828). Bruno Mégret est l'entrepreneur politique. Son objectif est de donner une direction au FN. Son engagement est la caisse de résonance des idées environnementalistes de la Nouvelle Droite de l'innovateur idéologique qu'est Alain de Benoist. II ne serait pas juste, cependant, de dire que le FN était silencieux sur l'environnement avant 1990 (cf. figure 1). Son environnementalisme à l'époque était chargé de référence à l'idée de la France éternelle, du fait que "la terre ne ment pas » du maréchal Pétain. L'environnementalisme anti-lumière ou réactionnaire irrigue ainsi l'extrême droite. Chez Marine Le Pen, l'entrepreneur idéologique fut plutôt Laurent Ozon, qui milite pour la relocalisation de l'économie et le régionalisme (François, 2016, p. 190). L’idéologie environnementaliste est ici celle de la production et de la vente de produits bio "sains et enracinés ${ }^{29}$. Elle développe les vieux thèmes des campagnes vertueuses contre les villes vicieuses, de la centralité de Paris contre les pouvoirs locaux des petites villes et des campagnes et évidemment le refus

28 François, S. (2016), "L'extrême droite française et l'écologie. Retour sur une polémique ", Revue française d'histoire des idées politiques, 44(2), 187-208.

29 Voir le site de vente en ligne "Au Bon sens". de la mondialisation. La conséquence de cet environnementalisme nationaliste est l'apologie des réseaux de distribution courts, de l'enracinement, de l'agriculture traditionnelle et du « manger local, penser global ". L'environnement est en quelque sorte instrumentalisé pour lutter contre la mondialisation des échanges, mais aussi les flux migratoires. Tout le discours régionaliste, antimondialisation et anti-banlieue (civilisation urbaine) est repris dans une perspective nationaliste. Les idées de la Nouvelle Droite ont ainsi trouvé dans l'activité politique du Front National un véhicule de diffusion de leurs idées.

Le Parti Communiste a été très tôt (cf. figure 1) un outil de transmission des idées de l'écologie politique. L’historien des idées communistes Pascal Acot (1988) rappelle ces liens dans son Histoire de l'écologie ${ }^{30}$. La relation environnementcommunisme n'est cependant pas toujours la même d'une période à l'autre. Le Parti Communiste est pro-nucléaire. La supériorité du socialisme sur le capitalisme, dit Maurcie Thorez, est illustrée par l'existence d'une centrale nucléaire civile en Union soviétique ${ }^{31}$. Il est aussi parmi les premiers à quantifier les coûts économiques de la dégradation de l'environnement et des milieux naturels ${ }^{32}$. Alors que l'environnement est un instrument pour lutter contre la mondialisation et l'immigration à l'extrême droite, il est un instrument pour lutter contre le capitalisme à l'extrême gauche communiste.

La destruction de l'environnement était une raison de plus - à côté des inégalités

\footnotetext{
30 Acot, P. (1988), Histoire de l'écologie, Paris: PUF. 31 Buton, P. (2016), "La gauche et la question écologique ", op. cit., p. 65.

32 Quin, C. (1972), Ce que coûte le capitalisme à la société, Paris : Économie et Politique.
} 
et du rapport d'exploitation qu'imposait un tel système - de croire en l'alternative communiste et en la possibilité de substituer au marché une économie dirigée et construite sur l'expertise technique et scientifique. Ainsi, le programme du PCF de 1987, "Justice, Liberté et Paix ", proposait aux électeurs " l'utilisation pleine et entière des progrès scientifiques et techniques, un cadre de vie de qualité, la protection et la mise en valeur de l'environnement et le retour au "produire français" "33. Il militait pour le protectionnisme afin de rapprocher le producteur du consommateur. Les grands leaders communistes comme Robert Hue, candidat à l'élection présidentielle de 2002, ou Marie-George Buffet, développent un discours pro-écologie en soutenant l'idée que le développement de la planète doit être durable et solidaire ${ }^{34}$. Le rappel de ces quelques faits le confirme et donne un contenu à l'environnementalisme de l'extrême gauche: une forme marxiste d'écologie politique ${ }^{35}$.

Les autres mouvements politiques à se revendiquer d'une forme d'écologie politique sont évidemment tous les partis que l'on peut appeler de la mouvance verte. Ce sont des partis de niche qui développent un discours alternatif qui oscille finalement entre la gauche, la droite et le " ni gauche ni droite ". S'ils ont généralement une posture libertaire ${ }^{36}$, post-1968, et un discours environnementaliste, ce

33 Prograrmme du PCF de 1987, "Justice, Liberté et Paix ".

34 Voir sur ce sujet les chroniques de Luc Foulquier, écologue et membre du Parti communiste français, notamment "Communisme et écologie, une longue histoire ", La Revue du Projet, 14, 11 mars 2011.

35 Biolat, G. (1973). Marxisme et environnement, Paris Édition sociale.

36 Villalba, B. (2011), «La transmutation d'Europe Écologie-Les Verts", in P. Bréchon (dir.), Les dernier diffère. L'histoire des grandes figures de l'écologie politique et des partis verts en sont la preuve. La candidature de René Dumont à la présidentielle de 1974 se place dans ce l'on pourrait appeler une sorte de socialisme associationniste renouvelé. Il s'agit d'un scientifique, agronome, qui décide de porter son discours sur la place publique. Yves Cochet est quant à lui proche de la théorie de la décroissance, parce qu'il estime que l'option de la décroissance n'est pas une question de choix, mais qu'elle s'imposera dans les faits ${ }^{37}$. Brice Lalonde a un parcours intellectuel complexe. Il est antinucléaire à ses débuts, libertaires, puis est très proche d'une forme d'environnementalisme étatique, pour finir, à la fin de son parcours politique, dans les années 1990, proche de l'écologie de marché lorsqu'il se rapproche du parti Démocratie Libérale d'Alain Madelin.

Dominique Voynet fut elle aussi ministre d'un gouvernement de gauche, portant l'idée que l'écologie peut renouveler en profondeur le discours de la gauche. Il s'agit d'imposer l'idée que la gauche peut renouveler sa critique du capitalisme et reconstruire un idéal en se fondant sur une forme ou une autre d'écologie politique.

À ces quatre figures de l'écologie s'ajoute Corinne Lepage qui fut ministre de l'Environnement d'un gouvernement de droite, et ajoute ainsi à une forme de confusion. Son mouvement politique Cap21 s'est rattaché au Mouvement Démocrate (MODEM) de François Bayrou en 2002 et a défendu le principe de vérité des coûts écologiques. Il s'est placé ainsi dans le cadre de la théorie des coûts sociaux. Cela

partis politiques français (chapitre 5, ici p. 151), Paris : La Documentation française.

37 Ibid., p. 133. 
Tableau 1 : Chronologie des partis politiques écologiques de 1970 à 2016

\begin{tabular}{|c|c|c|c|}
\hline $1970-1980$ & 1980-1990 & $1990-2000$ & $2000-2016$ \\
\hline $\begin{array}{l}\text { 1973, Écologie } \\
\text { et Survie, } \\
\text { Antoine } \\
\text { Waechter et } \\
\text { Solange Fernex } \\
\text { 1974, Comité de } \\
\text { soutien à René } \\
\text { Dumont } \\
\text { 1974-1983. Les } \\
\text { Amis de la Terre } \\
\text { se transforme } \\
\text { en parti : } \\
\text { Le collectif } \\
\text { écologique } \\
\text { (1978) } \\
\text { 1979, Europe } \\
\text { Écologie }\end{array}$ & $\begin{array}{l}\text { 1981, Aujourd'hui } \\
\text { l'écologie, Brice } \\
\text { Lalonde, Amis de la } \\
\text { Terre } \\
\text { 1981, Confédération } \\
\text { écologiste } \\
\text { 1982, Mouvement } \\
\text { d'écologie politique, } \\
\text { Philippe Lebreton puis } \\
\text { Parti écologiste (PE) } \\
\text { 1984, Les Verts, fusion } \\
\text { de PE et CE } \\
\text { 1986, Antoine } \\
\text { Waechter adopte le } \\
\text { "ni droite ni gauche " } \\
\text { \& 1984 Convergence } \\
\text { écologie, Brice } \\
\text { Lalonde, libertaire et } \\
\text { démocrate. } \\
\text { 1988-1998 Alternative } \\
\text { rouge verte, Pierre } \\
\text { Juquin, ancien } \\
\text { communiste }\end{array}$ & $\begin{array}{l}\text { 1990, Génération Écologie } \\
\text { (GE), Brice Lalonde, position } \\
\text { à gauche jusqu'en } 1994 \\
\text { 1993, Entente Écologiste } \\
\text { (GE + Les Verts) } \\
\text { 1994, Mouvement écologiste } \\
\text { indépendant (Antoine } \\
\text { Waechter) } \\
\text { 1994, Écologie Bleue } \\
\text { 1994, Confédération des } \\
\text { verts indépendants } \\
\text { 1994, Les Verts, Dominique } \\
\text { Voynet, alliance avec la } \\
\text { gauche, gauche plurielle } \\
\text { 1996 } \\
\text { 1997, Écologie citoyenne } \\
\text { 1994, Convergence } \\
\text { Écologie Solidarité, Noël } \\
\text { Mamère - CES }\end{array}$ & $\begin{array}{l}\text { Alliance Écologiste } \\
\text { Indépendante, } \\
\text { Jean-Marc } \\
\text { Governatori } \\
2008 \text { Europe } \\
\text { Écologie } \\
\text { Europe Écologie les } \\
\text { Verts (2010-2015) } \\
\text { 2015, Union des } \\
\text { démocrates et } \\
\text { des écologistes, } \\
\text { Parti écologiste de } \\
\text { François de Rugy } \\
\text { + Front Démocrate } \\
\text { de Jean-Luc } \\
\text { Benhamias (2014) } \\
\text { + Génération } \\
\text { Écologie } \\
\text { 1991-2016) }\end{array}$ \\
\hline
\end{tabular}

Source: Villalba, B. (2011), "La transmutation d’Europe Écologie-Les Verts ", in P. Bréchon (dir.), Les partis politiques français (chapitre 5), Paris : La Documentation française.

explique pourquoi il est admis que l'idéologie des Verts et de Génération Écologie est finalement relativement indéfinie ${ }^{38}$.

L'histoire des partis verts est celle d'une multitude de structures éphémères qui se mettent en place à la veille de chaque échéance électorale pour se dissoudre une fois la campagne achevée ${ }^{39}$. C'est l'histoire de partis qui ont du mal à trouver leurs marques sur l'échiquier politique français et peinent à s'incarner et à se structurer en un parti homogène pérenne. Ainsi, comme le souligne Sébastien Repaire, "la pensée écologique dont les Verts sont les héritiers n'est pas une doctrine structurée et figée, mais présente tous les traits d'un

39 Repaire, S. (2015), "Les "archives des Verts" du Centre international de recherches sur l'écologie (CIRE) : documenter l'histoire proche de l'écologie politique ", Histoire@Politique,27(3), 146-161. 
corpus théorique fort varié ${ }^{40}$. Les nombreux changements de nom et la multitude des partis écologiques français en sont l'illustration comme le reflet des différents courants écologistes (cf. tableau 1 ). Cette difficulté provient pour partie d'un effet de génération, qui a opposé deux formes de militantisme, l'une des années 1970 axée sur la mobilisation, l'autre des années 1990 portée sur la professionnalisation ${ }^{41}$. Ce clivage découle directement de la première forme organisationnelle de l'écologie française, à savoir l'association les Amis de la Terre, fondée par Alain Hervé en 1970.

La difficulté éprouvée par le parti vert à s'insérer dans l'opposition droite-gauche détermine en partie la position des deux grandes forces de gouvernement que sont la droite républicaine et la gauche socialiste. Ces derniers cherchent à intégrer l'environnement pour limiter la concurrence politique et renforcer leur poids électoral. Ils cherchent aussi à proposer un discours sur l'environnement qui soit en phase avec le caractère généraliste de leurs propositions politiques et plus généralement leur goût pour le progrès économique et social.

La gauche socialiste soutient que le progrès économique est à l'origine du progrès social et que l'État peut agir sur le progrès économique pour aiguiller le progrès social. II a pourtant de 1981 à 1997 utilisé l'environnementalisme pour ne pas laisser se développer à sa gauche un discours alternatif qui pourrait lui nuire

40 Repaire, S. (2016), "La création des verts : une intégration idéologique de l'écologie politique?", Revue française d'histoire des idées politiques, 44(2), 93-125 (ici p. 102, note 40).

41 Ollitrault, S. (2001), "Les écologistes français, des experts en action ", Revue française de science politique, 51(1), 105-130. électoralement. Le Parti Socialiste, après le débat sur le "zégisme $»^{42}$ n’a pas, en effet, retenu le principe d'une croissance zéro qui l'aurait placé dans le camp de l'écologie politique. Il a pourtant verdi son discours: en 1991, le PS, sous l'influence du courant fabiusien qui occupe le poste de secrétaire à l'environnement presque de 1990 à 2011, adopte, en effet, le principe du développement durable ${ }^{43}$. Le modèle du développement durable permet de dire aux électeurs que l'on continue de défendre l'équité sociale, mais désormais cette recherche du développement dans l'équité doit aussi tenir compte de l'équilibre des systèmes naturels. L'environnementalisme des socialistes est donc interventionniste. Le pic de 1997 correspond à l'accord passé dès le premier tour des élections législatives de 1997 entre le Parti Socialiste et les Verts. Cette majorité dite plurielle supposait un verdissement plus conséquent du programme du parti. Elle prend fin en 2002, date où le PS réduit fortement la part qu'il laisse à l'environnement dans son programme. En 2007 et 2012, le PS et les Verts tentent à nouveau de passer des accords, ce qui suppose une sorte de convergence programmatique a minima qui peut expliquer ce nouveau verdissement.

La droite républicaine, c'est-à-dire l'union des gaullistes et de la droite classique, a un rapport à la question environnementale chaotique ${ }^{44}$. Elle a été à l'origine du premier ministère de l'Environnement en

42 Défense de la croissance zéro.

43 Duverger, T. (2011), Le Parti socialiste et l'écologie, 1968-2011, Paris: Fondation Jean Jaurès.

44 Sainteny, G. (1999), "La droite française et l'écologisme ", Commentaire, 21, p.1049-1062; Persico, S. (2016), "Déclarer qu’on va protéger la planète, ça ne coûte rien. Les droites françaises et l'écologie (1971-2015)", Revue française d'histoire des idées politiques, 44(2), 157-186 (ici p. 159). 
1973, mais a entre 1978 et 1988 été quasi muette sur les questions environnementales (cf. figure 1). De 1988 à 2007, c'est le moment de l'environnementalisme de la droite républicaine. Cette droite républicaine est plus favorable au capitalisme que la gauche et fonde sa culture politique sur des valeurs et des croyances conservatrices. Elle a accompagné les Trente Glorieuses et son idéologie du progrès économique et social par la croissance de la production et de la consommation. Son environnementalisme est très éloigné de toute forme de critique du progrès économique. Il n'a pas en ce sens de lien avec l'écologie politique. L'environnementalisme n'est pas non plus une forme de développement durable, car dans les années 1970 ses principales mesures sont des mesures de protection de la nature. L'environnementalisme ce n'est pas un équilibre, mais une forme de conservatisme. La droite met en place le Conservatoire du littoral, la loi sur la protection de la nature, la loi sur les déchets, etc. De 1988 à 2007, cette forme d'environnementalisme protectionniste et conservateur est abandonnée avec l'action partisane de grandes figures de l'écologie à droite: Michel Barnier pour les années 199045, Patrice Hernu du mouvement l'Écologie Bleue (mouvement créé en 2002) et Nathalie Kosciusko-Morizet à partir de 2004. Ces figures de l'environnementalisme de droite ont ainsi importé le principe du développement durable dans les programmes de la droite en lui donnant, cependant, une dimension moins sociale et plus environnementale. Plus fidèle, en ce sens, à l'environnementalisme de la protection des années 1970. Les engagements de Michel Barnier sont

45 Persico, S. (2016), ibid., pp. 168-169, note 45. très proches de la théorie du développement durable. La présidence Sarkozy inaugure un ministère du Développement durable avec Nathalie Kosciusko-Morizet. L’Écologie Bleue défend aussi le développement durable ${ }^{46}$, mais avec un principe de performance qui sera repris par Nathalie Kosciusko-Morizet. La conséquence de cette conversion est la défense par la droite d'une écotaxe qui est l'une des mesures phares de l'environnementalisme technocratique de l'école néoclassique standard.

La première conséquence de cette évolution est la domination presque sans partage de l'idéologie du développement durable sur l'échiquier politique et la faiblesse de l'écologie politique et de l'environnementalisme anti-lumière qui ne semblent ni l'un ni l'autre pouvoir rivaliser avec cette idée que la croissance économique doit désormais tenir compte de ses conséquences environnementales.

La seconde conséquence est plus méthodologique. Toutes ces filiations montrent que l'introduction des différentes formes d'environnementalisme dans les programmes politiques des grands partis dépend du regard d'un entrepreneur politique et d'un principe de cohérence idéologique. À chaque fois on peut, en effet, désigner par leur nom les hommes ou les femmes qui ont poussé la thématique environnementale de leurs partis. Cela signifie que le poids de l'item environnement dans le programme politique de chaque parti dépend principalement de l'engagement des porteurs du projet environnemental dans chaque parti. L'apport de la théorie de l'entrepreneur tient ainsi sa promesse de pouvoir décrire

46 www.ecologiebleue.eu 
et expliquer sans recourir à une forme ou l'autre de déterminisme la part plus ou moins forte dans un programme politique d'une thématique aussi médiatique que l'environnement. L'autre point intéressant est qu'il y a bien un déterminisme idéologique au sens où chaque parti choisit l'environnementalisme qui ne remet pas en cause sa cohérence. Il y a une traçabilité des idées. L'écologie politique n'est pas portée par l'extrême droite et l'appel de la forêt par l'extrême gauche communiste. Cela indique que ce qui détermine les choix est un critère logique relevant de la méthode déductive et de l'argumentation.

Cet article n'a évidemment pas traité tous les aspects du verdissement du discours politique et in fine des politiques publiques. Il a permis, cependant, d'illustrer l'originalité d'une approche par la théorie de l'entrepreneur, autrement dit d'une explication qui donne toute sa place à la contingence contre l'analyse et s'écarte de la seule recherche de relations de nécessité. Il a aussi permis de rappeler le rôle central joué par le Parti Communiste dans la diffusion de l'écologie politique en France, d'une part, et la manière dont la transformation d'une innovation intellectuelle en innovation politique peut servir à populariser cette idée, d'autre part. Reste à décrire la manière dont les entrepreneurs intellectuels et/ou politiques manœuvrent pour mettre une fois élu et durant les élections la question de l'environnement à l'agenda politique. Mais aussi à compléter cette histoire par une étude fine des liens qui existent entre la transformation des représentations des membres de la société dans son ensemble et la production d'une innovation intellectuelle ${ }^{47}$. Il n'est pas impossible, en effet, que les entrepreneurs intellectuels ne fassent que traduire l'esprit d'une époque.

François Facchini, Professeur des Universités (Paris I Panthéon-Sorbonne), est spécialiste d'économie de la politique et d'économie du paysage. II est l'auteur de l'article «L'entrepreneur politique » dans le Dictionnaire économique de l'entrepreneur (Garnier, 2017) et de nombreux articles sur l'entrepreneur marchand dans des revues de sciences économiques.

Benjamin Michallet est économiste à la Banque centrale de Cayenne et doctorant à I'Université de Paris I Panthéon-Sorbonne. II a plus particulièrement travaillé sur l'économie politique des choix de politiques publiques en matière environnementale.

47 Cf. Quenet, G. (2014), Qu'est-ce que l'histoire environnementale?, op. cit., p. 256. 